\title{
A NUMERICAL APPROACH TO THE STANDARD MODEL OF WATER HAMMER WITH FLUID-STRUCTURE INTERACTION
}

\author{
SŁAWOMIR HeNCLIK \\ Institute of Fluid-Flow Machinery, Polish Academy of Sciences, Gdańsk, Poland \\ e-mail: shen@imp.gda.pl
}

\begin{abstract}
In the classic water hammer (WH) theory, 1D liquid flow in a quasi-rigid pipe is assumed. When the pipe is flexible or is fixed to the foundation with elastic supports, the dynamic fluid structure interaction (FSI) should be taken into account for more accurate modelling of the system behaviour. The standard model of WH-FSI for a straight pipe reach is governed by fourteen hyperbolic partial differential equations of the first order, two for 1D liquid flow and twelve for 3D motion of the pipe. This model is presented in the paper and an algorithm for its numerical solution based of the method of characteristics is proposed. Basic boundary conditions (BC) are shortly discussed. The important condition at the junction of two subpipes fixed to the foundation with a viscoelastic support is presented in details and a general method of its solution is proposed.
\end{abstract}

Keywords: water hammer, transient pipe flow, fluid-structure interaction, standard model, method of characteristics

\section{Introduction}

The water hammer (WH) phenomenon has been of scientific interest for over a century. Extensive studies on this effect can be found in the works of Wylie and Streeter (1993), Almeida and Koelle (1992), Adamkowski (2013) or Ghidaoui et al. (2005). WH is a result of a sudden change in pipe flow conditions due to valves operation, hydraulic machinery load variation or other reasons. During the transient the changes in liquid velocity result in pressure variations, which may travel along the pipe producing loads of the structure (pipe, supports, hydraulic devices) and perturbations to the system functioning or even its damage. WH can appear in various pipe flow systems like hydro-power installations, nuclear reactor cooling systems, engine fuel injection pipes and others. In some cases, the pipe is stiff and does not move, so the action of the liquid onto the pipe is considered as quasi-static. When the pipe is elastic or the whole piping can move on its supports, this motion forced by the unsteady flow, influences in reverse the flow variables, and the dynamic fluid-structure interaction (FSI) has to be taken into account. These effects has been examined by scientists for a few decades and the works of Wiggert et al. (1987), Tijsseling and Lavooij (1990), Wang and Tan (1997) or Wiggert and Tijsseling (2001) can be pointed as the reference. A specific and a complex area of potential application of WH-FSI concepts is the phenomenon of blood flow in human arteries, being an important area of research, discussed e.g. by Alastruey et al. (2012).

Three main factors responsible for FSI coupling are pointed in literature. The weakest is the friction between the pipe-wall and the liquid. Due to physics of WH-FSI, an unsteady friction model should be used for modelling of this effect, and one of the existed models used for classic WH, discussed e.g. by Adamkowski and Lewandowski (2006), Vitkovsky et al. (2006) or Urbanowicz and Zarzycki (2012) could be adopted. However, the quasi-steady model is still quite popular and often used because of its simplicity. The Poisson effect is responsible for the 
second FSI coupling factor. Pressure variations induce circumferential stresses and strains in the pipe-wall which are accompanied by longitudinal ones. The latter propagates along the pipe faster than the pressure wave in the liquid and produces in the reverse action additional pressure oscillations known as the precursor (PC) wave. The third and the strongest FSI mechanism is the junction coupling (JC) effect which appears at pipe bends, knees, ends, valves, reductions, etc. In fact, this phenomenon is especially important if the pipeline is able to move as a whole structure. Due to this motion, strong interaction between the pipe and the liquid variables can occur. The motion of the junction can be also responsible for coupling between different modes of structural vibrations of neighbouring sub-pipes. As far as the friction and Poisson couplings are modelled by certain terms in the governing equations, JC is modelled by boundary conditions (BC). The JC mechanism can also be responsible for producing the WH effect by sudden motion of the structure.

In the classic water hammer theory a stiff and immovable pipe with one dimensional (1D) liquid flow is assumed. FSI is considered to be a quasi-static effect and the pipe-wall elasticity is taken into account only within the formula for the celerity of elastic waves in the liquid. Two hyperbolic partial differential equations (PDE) of the first order are used for description of the liquid flow which allows one to find the pressure $p$ and velocity $v$ as a function of the position $x$ and time $t$. When the dynamic FSI is taken into account, two main approaches are proposed in the literature. The simpler consists of four PDEs, two for 1D liquid flow and the other two for the longitudinal motion of the straight pipe reach. These four hyperbolic equations form the four equations (4E) model of WH-FSI. In this model, the 1D approximation neglects the radial motion of the pipe and the liquid and is valid for low frequency assumption. When 3D motion of the pipe is possible, additional ten equations of lateral and torsional movement produce altogether the fourteen equations $(14 \mathrm{E})$ standard model of WH-FSI. For numerical modelling of the WH behaviour, the method of characteristics (MOC) is frequently used. When FSI effects are taken into account, the finite elements method (FEM) can be alternatively exploited to the structural equations, which produces the MOC-FEM technique. Pure FEM based approach are also proposed. On the other hand, the fully MOC based algorithm is proposed in the fundamental paper of Wiggert et al. (1987), however only general ideas of their method were presented there. A thorough presentation of the MOC approach is included in the work of Tijsseling (1993), who applied his model to a 2D pipeline system. In fact, for structural analyses, the FEM technique can be considered to be more suitable, however using a unified MOC approach to the liquid as well as to the structure is justified due to the wavy nature of the phenomenon.

In the current paper, the standard model of WH-FSI is described and a MOC based method of numerical solution for a $3 \mathrm{D}$ pipeline system is presented. The main steps of the algorithm are discussed and some details and novel approaches are especially explained. An important part of the method is the $\mathrm{BC}$ at the junction of two sub-pipes fixed to the foundation with a viscoelastic support, which is formulated as a differential equation of motion. The solution to this equation is found and presented in a compact form, convenient for numerical computations. The author does not know any other work where such a detailed 3D treatment of that problem is considered and solved.

\section{Assumptions and governing equations}

The coordinate system and variables used in the current approach are presented in Fig. 1.

The pipe of length $L$, wall thickness $e$, diameter $D$ is straight, prismatic, thin-walled $(e / D \ll 1)$ and slender $(D / L \ll 1)$. The pipe material is linearly elastic and no buckling appears. The flow velocity $v$ is of little relativity to the elastic waves celerity $c(v / c \ll 1)$. The liquid is weakly compressible, linearly elastic and its density changes are small (pressures are low relative to the bulk modulus $K, p / K \ll 1$ ). The low frequency assumption applied means 


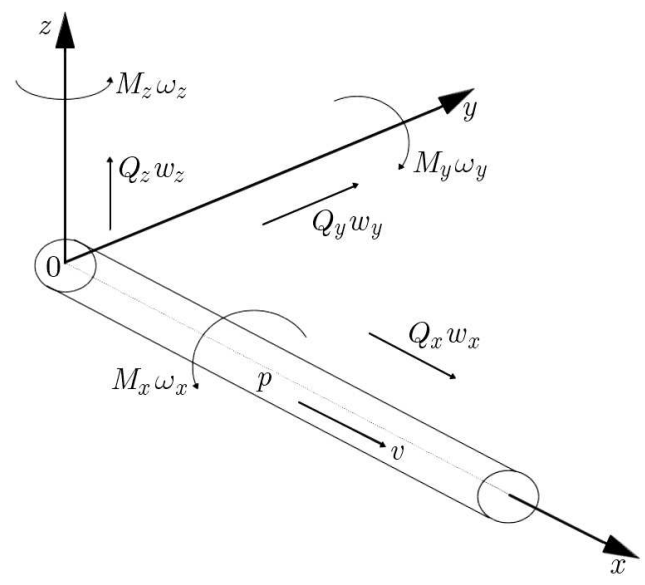

Fig. 1. Variables and coordinate system used for modelling of the WH-FSI phenomenon

neglecting of the radial inertia effects of the liquid and the pipe wall. The friction between the pipe and the liquid is taken into account and a quasi-steady model is assumed. The structural damping of the pipe material and two-phase flow are assumed not to be present. The motion of the system for a straight pipe reach is described by four uncoupled sets of PDEs. The first one consists of four equations for longitudinal motion of the system. The second set of two equations governs torsional waves in the pipe. Another two sets of four PDEs each define the lateral motion of the pipe in two perpendicular planes - vertical $(0 z x)$ and non-vertical $(0 y x)$. It is assumed that the liquid does not interact with the pipe in torsional vibrations. For the lateral movement, the Timoshenko beam model is used with the liquid being accounted as an added mass for linear motion. Liquid rotational inertia effects and the Coriolis force due to bending angular velocity are neglected.

Four equations govern the longitudinal motion of the system. Their detailed derivation can be found e.g. in Wang and Tan (1997). Two of the equations describe 1D liquid flow

$$
\frac{\partial v}{\partial t}+\frac{1}{\rho} \frac{\partial p}{\partial x}=-g \sin \alpha-\frac{4 \tau_{s}}{\rho D} \quad \frac{\partial v}{\partial x}+\frac{1}{\rho c^{2}} \frac{\partial p}{\partial t}=2 \nu \frac{\partial w_{x}}{\partial x}
$$

where $\nu$ is the Poisson coefficient and $\rho$ is liquid density. The pressure wave celerity $c$ is given as

$$
c=\sqrt{\frac{K}{\rho}} \frac{1}{\sqrt{1+\chi\left(1-\nu^{2}\right)}}
$$

The right-side factor in the formula above expresses the influence of pipe-wall elasticity. The parameter $\chi$ used in it is defined as

$$
\chi=\frac{K D}{E e}
$$

The other two equations of the $4 \mathrm{E}$ model govern the longitudinal pipe motion defined with velocity $w_{x}$ and stresses $\sigma_{x}\left(\sigma_{x}=Q_{x} / A_{s}, A_{s}\right.$ is the pipe cross-section area)

$$
\frac{\partial w_{x}}{\partial t}-\frac{1}{\rho_{s}} \frac{\partial \sigma_{x}}{\partial x}=-g \sin \alpha+\frac{\tau_{s}}{e \rho_{s}} \quad \frac{\partial w_{x}}{\partial x}-\frac{1}{\rho_{s} c_{s}^{2}} \frac{\partial \sigma_{x}}{\partial t}=-\frac{\nu D}{2 E e} \frac{\partial p}{\partial t}
$$

The pipe material density is $\rho_{s}$ and the longitudinal elastic waves travel in it with the celerity $c_{s}$

$$
c_{s}=\sqrt{\frac{E}{\rho_{s}}}
$$


The gravitational term ( $g$ is acceleration of gravity) depends on the angle $\alpha$ between the horizon and the pipe. One can easily identify the components responsible for the Poisson and friction couplings. For the current quasi-steady liquid pipe-wall friction model, the following formula is used for determination of the shear stresses $\tau_{s}$

$$
\tau_{s}=\frac{\lambda \rho}{8}\left(v-w_{x}\right)\left|v-w_{x}\right|
$$

In the equation above, $\lambda$ is the Darcy-Weisbach friction factor and should be adequately determined. The pipe torsional vibrations are governed by the standard two equations (like for the rod)

$$
\frac{\partial \omega_{x}}{\partial t}-\frac{1}{\rho_{s} I_{0}} \frac{\partial M_{x}}{\partial x}=0 \quad \frac{\partial \omega_{x}}{\partial x}-\frac{1}{G I_{0}} \frac{\partial M_{x}}{\partial t}=0
$$

where $M_{x}[\mathrm{Nm}]$ is torsional moment of the force, $\omega_{x}[1 / \mathrm{s}]-$ angular torsional velocity, $I_{0}\left[\mathrm{~m}^{4}\right]$ - pipe cross-section polar moment of inertia and $G[\mathrm{~Pa}]$ - shear modulus of the pipe material, $G=E /[2(1+\nu)]$. The shape of the above equations is the result of no-interaction assumption between the pipe and the liquid in torsional vibrations. However, if non-zero amount of accompanying water is going to be taken into account, then the factor $\rho_{s} I_{0}$ in Eq. $(2.7)_{1}$ should be adequately increased.

In the lateral motion at the non-vertical plane the governing equations for the Timoshenko beam model (Timoshenko and Young, 1955; Meirovitch, 1967) and the assumed coordinate system are found to be

$$
\begin{aligned}
\frac{\partial w_{y}}{\partial t}+\frac{1}{m} \frac{\partial Q_{y}}{\partial x} & =0 & \frac{\partial w_{y}}{\partial x}+\frac{1}{T} \frac{\partial Q_{y}}{\partial t} & =\omega_{z} \\
\frac{\partial \omega_{z}}{\partial t}-\frac{1}{b} \frac{\partial M_{z}}{\partial x} & =-\frac{1}{b} Q_{y} & \frac{\partial \omega_{z}}{\partial x}-\frac{1}{s} \frac{\partial M_{z}}{\partial t} & =0
\end{aligned}
$$

The variables above are the linear velocity $w_{y}[\mathrm{~m} / \mathrm{s}]$, shear force $Q_{y}[\mathrm{~N}]$, bending angular velocity $\omega_{z}[1 / \mathrm{s}]$ and the bending moment of force $M_{z}[\mathrm{Nm}]$. The parameters $m, b, s, T$ are introduced for more clear presentation of the model and the assumptions. They are defined in the following way

$$
m=\rho_{s} A_{s}+\rho A_{c}=\rho_{s} A_{s}(1+\xi) \quad T=\kappa G A_{s} \quad b=\rho_{s} I \quad s=E I
$$

The form of equations $(2.9)_{1}$ and $(2.9)_{3}$ results from the water-in-pipe accounting method - the water is taken into account in the former, not in the latter. Other possibilities can be tested by proper modelling of these parameters. In Wiggert et al. (1987) the expression for $b$ has included the water component as well and the current assumption was proposed by Tijsseling (1993). In fact, the influence of the weighted water component in $b$ can be numerically tested, calibrated and concluded. The parameter $\xi$ in $(2.9)_{1}$ expresses the ratio of the water-to-pipe mass and is given in

$$
\xi=\frac{\rho D}{4 \rho_{s} e}
$$

In Eq. $(2.9)_{3} I\left[\mathrm{~m}^{4}\right]$ is the axial moment of inertia of the pipe cross-section, and $\kappa$ in $(2.9)_{2}$ is the shear coefficient. A simple approach gives for a circular tube the value of $\kappa=0.5$, but due to more detailed analyses, slightly larger values are estimated. $\kappa=0.5+\nu /(8+6 \nu)$ was proposed by Cowper (1967) and $\kappa=0.5+\nu /(4+2 \nu)$ was estimated by Hutchinson (2001). In the vertical $0 x z$ 
plane, the equations are similar (the proper signs are the result of dextrose coordinate system) and the gravity term is also included

$$
\begin{aligned}
\frac{\partial w_{z}}{\partial t}+\frac{1}{m} \frac{\partial Q_{z}}{\partial x} & =-g \cos \alpha & \frac{\partial w_{z}}{\partial x}+\frac{1}{T} \frac{\partial Q_{z}}{\partial t} & =-\omega_{y} \\
\frac{\partial \omega_{y}}{\partial t}+\frac{1}{b} \frac{\partial M_{y}}{\partial x} & =\frac{1}{b} Q_{z} & \frac{\partial \omega_{y}}{\partial x}+\frac{1}{s} \frac{\partial M_{y}}{\partial t} & =0
\end{aligned}
$$

\section{Transformation of the equations}

\subsection{Longitudinal waves}

The fundamental step of the MOC technique is linear transformation of the governing equations to get $\mathrm{CE}$, which have the partial derivatives against $x$ and $t$ formed into absolute derivatives with time for a certain dependence $x(t)$. Such transformation applied to longitudinal equations (2.1) and (2.4) results in two sets of two equations each. They govern two coupled waves - WH and PC ones. The former propagates with the celerity $c_{0}$ (equations $\mathrm{C} 0$ ) and the equations $\mathrm{C} 1$ govern the $\mathrm{PC}$ wave which propagates with the celerity $c_{1}$. Because of the Poisson coupling, these celerities are slightly different than the original celerities $c$ and $c_{s}$ given with Eqs. (2.2) and (2.5). Moreover, these waves are not pure liquid and pure pipe waves as can be seen below. The $\mathrm{C} 0$ equations are

$$
\frac{d}{d t}\left(v+S w_{1}\right)+\varepsilon \frac{d}{d t}\left(r-\widetilde{S} q_{1}\right)=-(1+S) g \sin \alpha-(1-R) \frac{4 \tau_{s}}{\rho D}
$$

They are valid for the positive and negative characteristic slope $(\varepsilon= \pm 1)$ and the dependence $x(t)$ defined with $d x / d t=\varepsilon c_{0}$. The celerity of the $\mathrm{WH}$ wave is

$$
c_{0}=\frac{c}{\sqrt{A}}
$$

In Eq. (3.1), unified variables are used. The unified velocity $w_{1}$ is just the pipe section longitudinal velocity $w_{x}$. The unified pressure and longitudinal stresses are given by

$$
r=\frac{p}{\rho c_{0}} \quad q_{1}=\frac{\sigma_{x}}{\rho_{s} c_{1}}
$$

The following pair of equations $(\varepsilon= \pm 1)$ referred to as $\mathrm{C} 1$ governs the $\mathrm{PC}$ wave

$$
\frac{d}{d t}\left(R v-w_{1}\right)+\varepsilon \frac{d}{d t}\left(\widetilde{R} r+q_{1}\right)=(1-R) g \sin \alpha-(1+S) \frac{\tau_{s}}{\rho_{s} e}
$$

The characteristic slope is $d x / d t=\varepsilon c_{1}$ and the $\mathrm{PC}$ wave celerity $c_{1}$ is given as

$$
c_{1}=c_{s} \sqrt{A}
$$

The presented transformations are valid if the below relation holds

$$
1-\gamma+\chi>0
$$

where

$$
\gamma=\frac{K \rho_{s}}{E \rho}
$$


Relation (3.6) is valid for all practical cases, and for water in metal pipe and thin-walled assumption is even stronger $(>1)$. It is useful to define the following parameter $B$

$$
B=\frac{1}{2}\left[(1-\gamma+\chi)+\sqrt{(1-\gamma+\chi)^{2}+4 \gamma \chi \nu^{2}}\right]
$$

The correcting parameter $A$, which is slightly greater than $1(A=1$ for $\nu=0)$ can now be presented as

$$
A=1+\frac{\chi \nu^{2}}{B-\chi \nu^{2}}
$$

The Poisson coupling parameters $S, R$ are small $(R=S=0$ for $\nu=0)$ and defined below

$$
S=\frac{2 \gamma \nu}{B} \quad R=\xi S
$$

The tilde $S, R$ parameters are modified with the celerities ratio $\eta=c_{0} / c_{1}$ and given as

$$
\widetilde{R}=R \eta \quad \widetilde{S}=\frac{S}{\eta}
$$

The developed clear form of the $\mathrm{CE}$ has an important advantage as it allows for preliminary analyses of physical behaviour of the system even prior to computations. If the right sides of them are assumed zero (no friction and a horizontal pipe) and the variables in the parenthesis on the left sides are considered as new "wave variables" - velocities and stresses, these equations would represent simple elastic waves. But due to the shape of the wave variables they are not pure liquid $(\mathrm{WH})$ nor pure pipe (PC) waves. Thus the real physical quantities, velocities and stresses (pressures), are superpositions of the $\mathrm{WH}$ and $\mathrm{PC}$ waves. The dominant role is played by the former in liquid variables and the latter in the structure ones. These effects are possible to observe in experimental and numerical records (see Adamkowski et al., 2010 or Henclik, 2010).

\subsection{Torsional vibrations}

Transformation of governing equations (2.7) results in compatibility equations C4

$$
\frac{d w_{4}}{d t}-\varepsilon \frac{d q_{4}}{d t}=0
$$

They are valid for the characteristic slope $d x / d t=\varepsilon c_{4}$, and the celerity $c_{4}$ of torsional waves is

$$
c_{4}=\sqrt{\frac{G}{\rho_{s}}}
$$

The $\mathrm{CE}$ and unified variables, velocities $\mathrm{w}$ and loads $\mathrm{q}$, are numbered consequently with indexes 1, 2, 3 for linear and 4, 5, 6 for angular degrees of freedom, corresponding respectively to the $x$, $y, z$ axes. The variables in C4 equations are the normalized torsional angular velocity $w_{4}$ and the moment of force $q_{4}$

$$
w_{4}=h \omega_{x} \quad q_{4}=\frac{h M_{x}}{\rho_{s} I_{0} c_{4}}
$$

The parameter $h[\mathrm{~m}]$ introduced above to get the unified variables can be in fact arbitrarily selected. But for the model homogeneity, its value is assumed to be the same as calculated further for lateral motion. 


\subsection{Lateral motion}

Equations (2.8) of transversal movement in the non-vertical plane are transformed to $\mathrm{CE}$ representing shearing waves $\mathrm{C} 2$ coupled with bending waves C6. The equations $\mathrm{C} 2$ are given in

$$
\frac{d w_{2}}{d t}+\varepsilon \frac{d q_{2}}{d t}=\varepsilon \Omega w_{6}
$$

They are valid for the dependence $x(t)$ having the slope equal to the wave celerity $d x / d t=\varepsilon c_{2}$

$$
c_{2}=\sqrt{\frac{T}{T m}}
$$

The parameter $\Omega$ on the right side of (3.15) is a result of specific normalization of the equations and is explained further in $(3.20)_{2}$. The C6 equations have the following form

$$
\frac{d w_{6}}{d t}-\varepsilon \frac{d q_{6}}{d t}=-\Omega q_{2}
$$

They are valid for $d x / d t=\varepsilon c_{6}$, where

$$
c_{6}=\sqrt{\frac{s}{b}}
$$

The unified variables are

$$
w_{2}=w_{y} \quad q_{2}=\frac{Q_{y}}{m c_{2}} \quad w_{6}=h w_{z} \quad q_{6}=\frac{h M_{z}}{b c_{6}}
$$

The compact form of the lateral CE has been achieved by specific selection of the constant $h$ to keep the same scaling coefficient $\Omega[1 / \mathrm{s}]$ on the right side of them. The result of this procedure is

$$
h=\sqrt{\frac{b}{m}} \quad \Omega=\sqrt{\frac{T}{b}}
$$

Estimation of expression $(3.20)_{1}$ for the water in a metal pipe gives a value of about $D / 4$ for $h$.

In the vertical plane, we get C3/C5 coupled CE. The shearing waves are governed by C3 equations

$$
\frac{d w_{3}}{d t}+\varepsilon \frac{d q_{3}}{d t}=-g \cos \alpha-\varepsilon \Omega w_{5}
$$

They are valid as usual for the dependence $x(t)$ being the path of the wave that propagates with the same celerity as in the $\mathrm{C} 2$ case $\left(c_{3}=c_{2}\right)$. The bending waves $\mathrm{C} 5$ have the same celerity as in the $\mathrm{C} 6$ case

$$
\frac{d w_{5}}{d t}+\varepsilon \frac{d q_{5}}{d t}=\Omega q_{3}
$$

The definition of unified variables is analogous as in the C2-C6 case. 


\section{Numerical method}

The first step of the numerical algorithm is to integrate each CE in time within a specific time step $\Delta t$ to get finite difference equations which can be solved for subsequent time moments at the $x$ - $t$ plane. A proper construction of the numerical scheme is required. For stability and convergence, the CFL (Courant-Fredicks-Lewy) condition (Quarteroni and Valli, 1994; Almeida and Koelle, 1992), which determines the relation between the time step $\Delta t$ and space grid size $\Delta x$, is necessary

$$
C_{N}=\frac{c \Delta t}{\Delta x} \leqslant 1
$$

It is easy to fulfill the condition for one pair of $\mathrm{CE}$ (one wave). Keeping $C_{N}$ equal to one allows one to find the solutions without interpolation with the use of a scheme presented at the grid in Fig. 2 for the C4 torsional wave. Integrating (3.12) within the time $\Delta t_{4}$, we get a set of two linear equations

$$
w_{4}-q_{4}=b_{4 \mathrm{~L}} \quad w_{4}+q_{4}=b_{4 \mathrm{R}}
$$

The "history parameters" $b_{4}$ at the right side are a result of integrating the CE, and are given in

$$
b_{4 \mathrm{~L}}=w_{4}^{(4 \mathrm{~L})}-q_{4}^{(4 \mathrm{~L})} \quad b_{4 \mathrm{R}}=w_{4}^{(4 \mathrm{R})}+q_{4}^{(4 \mathrm{R})}
$$

The initial values of variables are taken at the beginning of $\mathrm{C} 4$ left $(\mathrm{L})$ and right $(\mathrm{R})$ characteristics as it is presented at the right part of Fig. 2. Two kinds of average parameters are defined and used consequently within the model, half of the sum and half of the difference

$$
\bar{b}_{4}=\frac{b_{4 \mathrm{R}}+b_{4 \mathrm{~L}}}{2} \quad \stackrel{\circ}{b}_{4}=\frac{b_{4 \mathrm{R}}-b_{4 \mathrm{~L}}}{2}
$$

Now, the solutions to (4.2) at the final point $F$ (in Fig. 2) can be presented as

$$
w_{4}=\bar{b}_{4} \quad q_{4}=\stackrel{\circ}{b}_{4}
$$

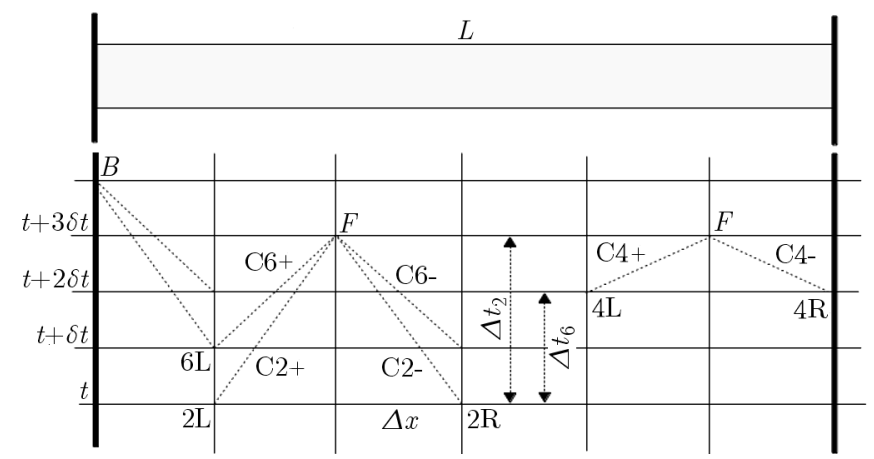

Fig. 2. A computational scheme for two coupled waves $\mathrm{C} 6 / \mathrm{C} 2$ of the celerity ratio 3:2 (at the left) and for a single wave $\mathrm{C} 4$ of the time step $\Delta t_{4}=\delta t$ (at the right)

A more complex case is for two coupled waves that propagate with different celerities, which is illustrated at the left part in Fig. 2 for the C2-C6 equations. If the ratio of shearing and bending waves celerities is a rational number, we can integrate each pair of $\mathrm{CE}(\mathrm{C} 2 / \mathrm{C} 6)$ within a specific time step $\Delta t,\left(\Delta t_{2}\right.$ and $\Delta t_{6}$ respectively $)$, which is an integer multiplication of the elementary step $\delta t$. 
In Fig. 2, the celerity ratio is $3: 2$ so the time step ratio is $2: 3$. This construction produces the same space step $\Delta x$ for both waves and it is possible to move across the $x$ - $t$ plane and to solve the equations for the unknown variables at each node without interpolation, as we can always find the starting point in the past for any characteristics. Small modifications are required at the beginning moments, but then we can start the characteristic at any position $x$ of the $t=0$ line due to the known initial conditions. As an alternative, a numerical scheme with interpolation can be also considered. After integration, the following set of four linear equations for the unknown variables is found

$$
\begin{array}{ll}
w_{2}+q_{2}-\mu_{2} w_{6}=b_{2 \mathrm{~L}} & w_{2}-q_{2}-\mu_{2} w_{6}=b_{2 \mathrm{R}} \\
w_{6}-q_{6}+\mu_{6} w_{2}=b_{6 \mathrm{~L}} & w_{6}+q_{6}-\mu_{6} w_{2}=b_{6 \mathrm{R}}
\end{array}
$$

In the integration process, the mean values of the right-hand side variables in (3.15) and (3.17) are assumed to be the arithmetic averages of their initial and final values, which produces the following form of $\mu$

$$
\mu_{i}=\frac{\Omega \Delta t_{i}}{2}
$$

The formulas for the history parameters can be easily derived and will not be presented herein. Due to the coupling between the waves, the parameters $\mu$ are assumed to be upper bounded for the stable numerical solutions, which produces an upper limit for the space size $\Delta x$ of the grid mesh. An estimation made with the application of an iterative method (see Bjoerck and Dahlquist, 1974) to partially transformed equations (4.6) has given for it a value of about $D / 2$. With slightly different analyses, Tijsseling (1993) suggested that the limit for an empty pipe should be $D /(2 \sqrt{2})$. In fact, the real space step will be selected with numerical tests. The solutions to (4.6) are

$$
w_{2}=\bar{b}_{2} \quad q_{2}=\frac{-\stackrel{\circ}{b}_{2}+\mu_{2} \bar{b}_{6}}{1+\mu_{2} \mu_{6}} \quad w_{6}=\frac{\bar{b}_{6}+\mu_{6} \stackrel{\circ}{b}_{2}}{1+\mu_{2} \mu_{6}} \quad q_{6}=\stackrel{\circ}{b}_{6}
$$

An analogous procedure can be applied for lateral equations C3/C5 in the vertical plane. For longitudinal motion, the results are calculated in a similar way, though a comment is necessary as the friction terms on the right-hand side of Eq. (3.1) and (3.4) are non-linear. Usually, a linearization scheme is proposed and a direct method of solution is used. However, the non-linear friction terms are small, so at the current approach they will be left within the history parameters and assumed as fixed values with the possibility of iterative improvement. One additional iteration is usually enough. Such a solution shows the advantage of using the history parameters. It is a convenient idea as the results do depend only on those parameters and they can be defined within a numerical routine in a different way for any changes in the model. Introducing material damping, changing the pipe-wall friction model or using interpolation only influences the shape of those parameters. The $\mathrm{C} 0 / \mathrm{C} 1$ history parameters include also the gravitational term $(\mathrm{C} 3 / \mathrm{C} 5$ ones as well). Finally, the solutions to $\mathrm{C} 0 / \mathrm{C} 1$ are

$$
v=\frac{\bar{b}_{0}+S \bar{b}_{1}}{1+R S} \quad w_{1}=\frac{-\bar{b}_{1}+R \bar{b}_{0}}{1+R S} \quad r=-\frac{\stackrel{\circ}{b}_{0}+\widetilde{S} b_{1}}{1+R S} \quad q_{1}=\frac{-\stackrel{\circ}{b}_{1}+\widetilde{R} b_{0}}{1+R S}
$$

Each group of equations is solved independently, but the same elementary time step $\delta t$ is necessary for all of them to keep the effectiveness of the method. This is because the coupling between variables of different groups may occur at boundaries, thus common time nodes are required there. The common space nodes at the boundaries can be also useful. In this case, the ratios of all the wave celerities should be rational numbers. Such a condition is always true 
within a certain precision, but the algorithm is effective if the integers for these ratios are not too large, so a wave-speed adjustment can be used by slightly changing the selected material or geometrical parameters of the system. This procedure is also used in other approaches, and the current author has developed and tested an algorithm allowing one to adjust all the five celerities of the elastic waves.

\section{Boundary conditions}

The piping is modelled as a set of straight pipes connected at junctions where certain relations are valid as the boundary conditions. In a more general case, some working elements may be also taken into account. The term non-pipe element (NPE) is used in Almeida and Koelle (1992) for them and they may be a valve, pump, turbine or other hydraulic device. NPE is assumed to be a rigid and massive element and can be fixed to the foundation with rigid or elastic supports (or staying unfixed). It is connected to individual pipes by a number of inlets-outlets for which hydraulic characteristics are determined. A simple example of NPE is a valve with its dependence between the pressure at the inlet at the left $\left(p_{\mathrm{L}}\right)$ and the outlet at the right $\left(p_{\mathrm{R}}\right)$ given as

$$
p_{\mathrm{L}}=p_{\mathrm{R}}+\frac{1}{2} \rho\left(v-w_{x}\right)^{2} \zeta(t)
$$

In the equation above, the loss factor $\zeta(t)$ is dependent on the valve opening degree, so it may change in time. For a complete valve closure, it becomes infinity what in fact changes the BC to have the form $v=w_{x}$. NPE will not be considered within the current model, however the junction is in fact a simple NPE of a constant and small loss factor. The mass and size of the junction can usually be neglected, but the general case can be analyzed as well. Using the current assumptions, the main dynamic liquid BC at the junction of two sub-pipes defines the pressure balance with a relation similar to (5.1). The condition is even simpler as the junction losses $\zeta$ are constant and usually small, which produces a possibility for iterative solution. A fundamental $\mathrm{BC}$ for the liquid is the continuity equation. For the FSI case and two sub-pipes at the junction, it has the following form

$$
v^{(\mathrm{L})}-w_{x}^{(\mathrm{L})}=v^{(\mathrm{R})}-w_{x}^{(\mathrm{R})}
$$

In spite of the fact that the junction is rigid, the velocities of the left and right edge of it used above are not, in general, the same as 3D motion is considered and the left and right pipe coordinate systems can be rotated (when the junction is a bend). The rigidity of the junction means it is rigid in itself and is rigidly fixed to the pipes. That is why the junction velocity $\mathbf{w}$ and pipe ends velocities $\mathbf{w}^{(\mathrm{L})}$ and $\mathbf{w}^{(\mathrm{R})}$ are uniquely related. In fact, they are identical for the dimensionless junction

$$
\mathbf{w}^{(\mathrm{L})}=\mathbf{w}^{(\mathrm{R})}=\mathbf{w}
$$

In this case, $\mathbf{w}$ is $6 \mathrm{D}$ velocity with $4,5,6$ components being angular velocities multiplied by $h$ defined in $(3.20)_{1}$. The above is a vector equation, so when using its representation in a coordinate system, proper transform matrices of directional cosines $\mathbf{U}^{(\mathrm{L})}, \mathbf{U}^{(\mathrm{R})}$ have to be applied. If the junction size is not negligible, the above condition has to be modified because linear motion of the junction edge (the pipe end) has a component being the result of the junction rotation as a rigid body.

The motion of the junction is dependent on the way it is fixed to the foundation. For a rigid fixing, there is no motion and the $\mathrm{BC}$ is $\mathbf{w}=\mathbf{0}$. When the junction is unfixed or fixed with an 


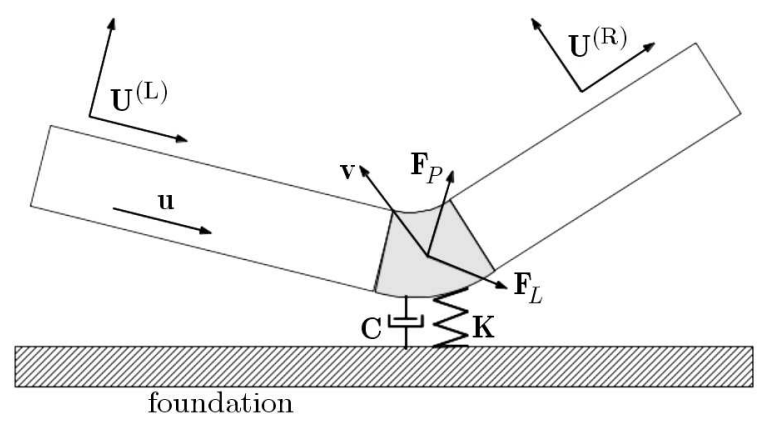

Fig. 3. Junction of two pipes fixed with a viscoelastic support

elastic support as it is presented in Fig. 3, the following junction equation of motion (EOM) should be formulated

$$
\mathbf{M} \ddot{\mathbf{u}}+\mathbf{C} \dot{\mathbf{u}}+\mathbf{K u}=\mathbf{F}_{L}+\mathbf{F}_{P}
$$

This is $6 \mathrm{D}$ equation for the junction displacement $\mathbf{u}$ having three linear $(1,2,3)$ and three angular $(4,5,6)$ coordinates. At the right-hand side of the EOM, there are pipe and liquid forces (and moments) acting onto the junction. On the left-hand side, there is an inertia term with the matrix $\mathbf{M}$ and the forces from the elastic support with stiffness and damping matrices $\mathbf{K}$ and $\mathbf{C}$. The Kelvin-Voigt model for the support reaction is assumed. In the EOM, the convention of multiplying angular $(4,5,6)$ coordinates in the vector $\mathbf{u}$ by the parameter $h$ is still valid and another one for normalization of the angular coordinates of the forces (the moments) by dividing them by $h$ is used as well. An adequate normalization is also done to the matrices $\mathbf{M}, \mathbf{K}, \mathbf{C}$ on the left-hand side. In general, determining these matrices is a separate task, but this problem will not be discussed herein. The EOM is simplified in special cases, e.g. for massless junction $\mathbf{M}=\mathbf{0}$, for zero damping $\mathbf{C}=\mathbf{0}$ and for not supported junction $\mathbf{K}=\mathbf{0}, \mathbf{C}=\mathbf{0}$.

The forces on the right side of the EOM depend on the flow and pipe motion variables. The liquid forces $\mathbf{F}_{L}$ are mainly the result of pipe static pressures and the pipe forces $\mathbf{F}_{P}$ appear explicitly in the CE, though proper transformations are required. Form (5.4) of the EOM allows one to understand the junction coupling mechanism, however it is not convenient for numerical solution. The right-hand side of the EOM can be transformed using the difference form of $\mathrm{CE}$ discussed in Section 4 to express the liquid and pipe forces as a linear function of the junction velocity $\mathbf{w}=d \mathbf{u} / d t$ in a new time instant. Finally, the EOM can be presented in the following form

$$
\widetilde{\mathbf{M}} \ddot{\mathbf{u}}+\widetilde{\mathbf{C}} \dot{\mathbf{u}}+\widetilde{\mathbf{K}} \mathbf{u}=-\mathbf{P} \mathbf{w}+\mathbf{a}
$$

In the above equation, the matrices on the left-hand side (with tilde) are the original ones divided by $\left(\rho_{s} A_{s} c_{s}\right)$. The essence of the method is the coupling matrix $\mathbf{P}$ which is symmetric and positive definite. It depends on the pipe-junction geometry and liquid-pipe material parameters. The junction history vector a depends on the previous values of the system variables at the junction and its neighborhood, and changes in each time step. Both formulas for the matrix $\mathbf{P}$ and vector a have been determined by the author to be applied in the algorithm. To find the solution to Eq. (5.5), its left-hand side has to be transformed to a finite difference form. Applying the Newmark method to it allows one to find the junction velocity $\mathbf{w}$ in a new time instant (the variables with "0" indexes are taken at the previous time instant)

$$
\mathbf{w}=\left(\frac{2}{\Delta t} \widetilde{\mathbf{M}}+\mathbf{P}+\widetilde{\mathbf{C}}+\frac{\Delta t}{2} \widetilde{\mathbf{K}}\right)^{-1}\left[\mathbf{a}+\widetilde{\mathbf{M}}\left(\frac{2}{\Delta t} \dot{\mathbf{u}}_{0}+\ddot{\mathbf{u}}_{0}\right)-\widetilde{\mathbf{K}}\left(\mathbf{u}_{0}+\frac{\Delta t}{2} \dot{\mathbf{u}}_{0}\right)\right]
$$

The matrix in the brackets is constant for the junction and positive definite, thus the equation can be easily solved in each time step. Knowing the velocity w, all other variables can be found. 


\section{Summary}

The water hammer phenomenon with dynamic fluid-structure interaction is discussed in the paper. The standard mathematical model of WH-FSI and an algorithm for its numerical solution on the basis of the MOC technique is presented. The fourteen governing equations are transformed into compatibility equations developed in a convenient, compact form with the use of "unified variables" - velocities and loads. The main steps and ideas of the numerical method are discussed. The concept of using "history parameters" allows one to effectively design the algorithm. The numerical scheme uses a properly designed computational grid exploiting the technique of wave-speed adjustment but the scheme with interpolation is also possible. An important part of the algorithm is the boundary condition at the junction fixed to the foundation with a viscoelastic support. It is formulated as a differential equation of motion, transformed and effectively solved with the use of a special coupling matrix and the Newmark method. A similar approach has been applied to the 1D 4E model and it works correctly. The algorithms are now being implemented in a computer code, and the numerical results will be compared with the records from experiments which are planned for the real 3D pipeline system built in the laboratory.

\section{Acknowledgements}

The results presented in the paper have been partially developed within research project No. N N504 478839 funded by the Ministry of Science and Higher Education of Poland.

\section{References}

1. Adamkowski A., 2013, Liquid Unsteady Flows in Closed Conduits (in Polish), Publishing House of the Institute of Fluid-Flow Machinery, Gdansk

2. Adamkowski A., Henclik S., Lewandowski M., 2010, Experimental and numerical results of the influence of dynamic Poisson effect on transient pipe flow parameters, 25th IAHR Symposium on Hydraulic Machinery and Systems, Timisoara, Institute of Physics Conference Series: Earth and Environmental Sciencees, DOI: 10.1088/1755-1315/12/1/012041

3. AdAMKowski A., LEWANdowski M., 2006, Experimental examination of unsteady friction models for transient pipe flow simulation, ASME Journal of Fluids Engineering, 128, 11

4. Alastruey J., Parker K.H., Sherwin S.J., 2012, Arterial pulse wave haemodynamics, Proceedings of the 11th International Conference on Pressure Surges, Lisbon, 401-442

5. Almeida A.B., Koelle E., 1992, Fluid Transients in Pipe Networks, Computational Mechanics Publications, Boston, London

6. BJoerck A., Dahlquist G., 1974, Numerical Methods, Prentice Hall, chap. 6.9.1 (Polish translation, 1987, PWN, Warsaw)

7. Cowper G.R., 1966, The shear coefficient in Timoshenko's beam theory, Journal of Applied Mechanics, 33, 6, 335-340

8. Ghidaoui M., Ming Zao, Mcilnnis D., Axworthy D., 2005, A review of water hammer theory and practise, Applied Mechanics Reviews, 58, 1, 49-76

9. Henclik S., 2010, Mathematical model and numerical computations of transient pipe flows with fluid-structure interaction, Transactions of the Institute of Fluid-Flow Machinery, 122, 77-94

10. Hutchinson J.R., 2001, Shear coefficients for Timoshenko beam theory, Journal of Applied Mechanics, 68, 1, 87-92

11. Meirovitch L., 1967, Analytical Methods in Vibrations, The Macmillan Company, New York 
12. Quarteroni A., Valli A., 1994, Numerical Approximation of Partial Differential Equations, Springer-Verlag, Berlin

13. Tijsseling A.S., Lavooij C.S.W., 1990, Waterhammer with fluid-structure interaction, Applied Scientific Research, 47, 273-285

14. TiJsseling A.S., 1993, Fluid - structure interaction in case of waterhammer with cavitation, PhD thesis, Delft University of Technology, Report 93-6, Faculty of Civil Engineering, http://www.win.tue.nl/ atijssel/pdf_files/Tijsseling_1993.pdf

15. Timoshenko S., Young D.H., 1955, Vibration Problems in Engineering, Nostrad Co. Inc., NY

16. Urbanowicz K., ZarzyCKi Z., 2012, New efficient approximation of weighting function for simulations of unsteady friction losses in liquid pipe flow, Journal of Theoretical and Applied Mechanics, 50, 2, 487-508

17. Vitkovsky J.P., Bergant A., Simpson A.R., Lambert M.F., 2006, Systematic evaluation of one-dimensional unsteady friction models in simple pipelines, Journal of Hydraulic Engineering, 132, 7, 696-708

18. WANG Z.M., TAN S.K., 1997, Coupled analysis of fluid transient and structural dynamic responses of a pipeline system, Journal of Hydraulic Research, 35, 1, 119-131

19. Wiggert D.C., Hatfield F.J., Stuckenbruck S., 1987, Analysis of liquid and structural transients in piping by the method of characteristics, ASME Journal of Fluids Engineering, 109, $161-165$

20. Wiggert D., Tijsseling A., 2001, Fluid transients and fluid-structure interaction in flexible liquid-filled piping, Applied Mechanics Reviews, 54, 455-481

21. Wylie E.B., Streeter V.L., 1993, Fluid Transients in Systems, Prentice-Hall, NJ 